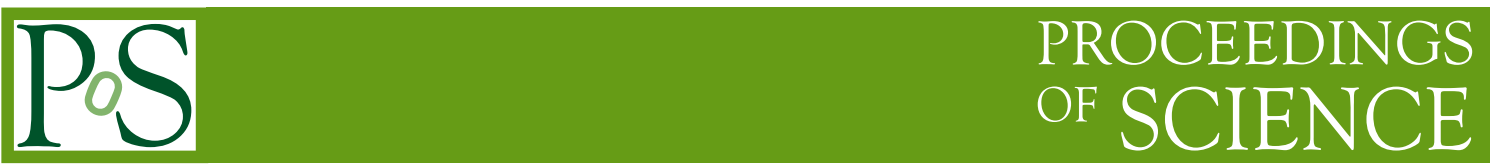

\title{
Cosmic Accelerators: New surprises in the CTA Era
}

\section{P.J. Meintjes*}

Department of Physics, University of the Free State, PO Box 339, Bloemfontein, South Africa

E-mail: MeintjPJ@ufs.ac.za

The Cherenkov Telescope Array (CTA) will provide a paradigm shift in terms of sensitivity and angular resolution, combined with a wide field of view (fov) for rapid survey work. The CTA threshold energy for detecting atmospheric Cherenkov showers will overlap with the Fermi-LAT energy regime, which provides an unique opportunity for detailed combined studies of gammaray pulsar emission as well as potential gamma-ray burst (GRB) sources. Apart from detailed studies of neutron star pulsars, pulsar wind nebulae and extra galactic sources like blazars, the unique sensitivity of CTA may provide the opportunity to study weaker emitters like cataclysmic variables. Of particular interest may be spun-up white dwarfs that went through a period of high mass accretion in the past. It has been shown that these systems may possess the required energetics to accelerate charged particles like electrons and nuclei to relativistic energies, which may produce high energy gamma-ray emission, through processes like inverse Compton scattering and pion decay. Two examples are discussed, namely AE Aquarii and the newly discovered AR Scorpii.

Frontier Research in Astrophysics - II

23-28 May 2016

Mondello (Palermo), Italy

\footnotetext{
* Speaker.
} 


\section{Introduction}

Since the first detection of ionizing cosmic radiation, or Cosmic Rays (CR) by Victor Hess in the years 1912-1913, subsequent studies over a wide energy range reveal (e.g. [1] for an overview) a distinct power-law spectrum up to the highest energies around $\varepsilon \sim 10^{20} \mathrm{eV}$ (see Fig. 1). The differential energy spectrum extends as $\varepsilon^{-s}$ (s=2.6-2.7) from $\mathrm{GeV}$ energies to a few $\mathrm{PeV}$ (e.g. $\mathrm{PeV}$ $=10^{15} \mathrm{eV}$ ), after which it steepens slightly at a few PeV and then flattens at about an EeV (e.g. EeV $=10^{18} \mathrm{eV}$ ). Subsequently it is turning over and terminating at about $100 \mathrm{EeV}$ (e.g. [1]).

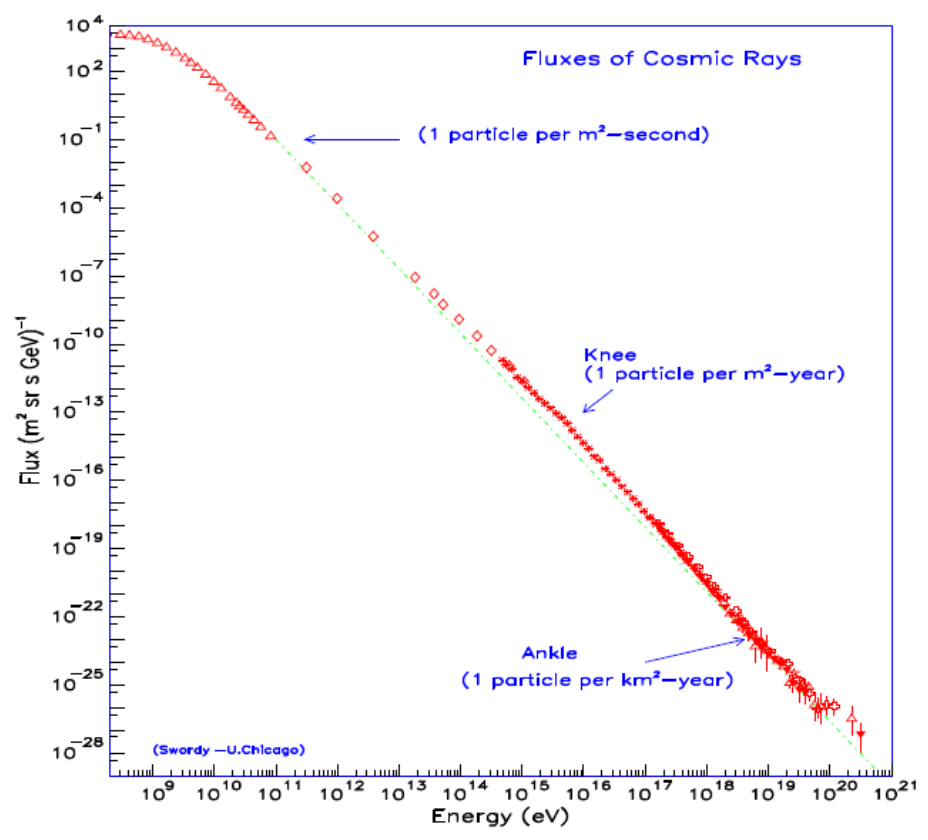

Figure 1: The measured cosmic ray spectrum. Adopted from [1].

This spectrum represents high energy particles produced through a plethora of processes under extreme conditions in various classes of astrophysical environments. The spectrum displays a bump between $\varepsilon \sim 10^{14}-10^{16} \mathrm{eV}$ (e.g. the "knee"). At energies below the knee the spectrum is believed to be produced mainly by galactic sources, e.g. pulsars, supernova remnants and pulsarwind nebulae to name a few, and above the knee, by extra-galactic sources like the central engines and jets of Active Galactic Nuclei (AGN). For example, CR arriving at Earth with energies of the order of $\varepsilon \sim 10^{20} \mathrm{eV}$ have a Larmor radius greater than the size of the Milky Way Galaxy, implying that they must have their origin in extreme environments beyond our Galaxy, and their arrival, measured by e.g. the Auger air shower array, correlates with nearby AGN (e.g. [2]). The power-law CR spectrum implies that these high energy cosmic messengers are not produced through thermal processes, but that the particles are accelerated through various processes, i.e. mainly shocks, in these exotic environments (e.g. [3] for a review).

The development of Cherenkov telescopes sensitive to gamma-rays with energies up to 100 $\mathrm{TeV}\left(\mathrm{TeV}=10^{12} \mathrm{eV}\right)$ can expand our understanding of the origin of high energy CR since gammarays are cosmic messengers that propagate through the universe from their place of origin without 
any deviation as a result of deflection off galactic and inter-galactic magnetic fields like the particle component of CR. Very High Energy (VHE) $\left(\varepsilon_{\gamma} \geq 10^{11} \mathrm{eV}\right)$ gamma-rays can be produced through various processes or channels involving high energy hadrons and leptons in astrophysical environments. For example, the main production channels of VHE gamma-rays through leptonic and hadronic processes are (e.g. [4]):

\section{Leptonic: Inverse-Compton Scattering}

Here VHE electrons up-scatter low energy photons over a broad energy range above the initial energy

$$
e_{\mathrm{VHE}}+\gamma_{\text {low energy }} \rightarrow e_{\text {lowenergy }}+\gamma_{\mathrm{VHE}}
$$

A source that contains a particle accelerator and a reservoir of low energy thermal photons, or photons produced by electrons radiating synchrotron radiation in magnetic fields, can be a significant source of gamma-rays through the inverse-Compton process.

\section{Hadronic: Pion Decay}

Accelerated protons or heavier nuclei interact with ambient protons, nuclei or photons in astrophysical environments, producing predominantly charged and neutral pions. Charged pions decay in a two-step process into electrons and two neutrinos, while neutral pions decay with $>99 \%$ probability into two gamma-rays. For proton-nucleus interactions one has

$$
\begin{aligned}
p_{\mathrm{VHE}}+\text { nucleus } & \rightarrow p^{\prime}+\pi^{ \pm}+\pi^{\circ} \\
\pi^{\circ} & \rightarrow 2 \gamma \\
\pi & \rightarrow \mu v_{\mu} \\
\mu & \rightarrow e v_{\mu} v_{\mathrm{e}}
\end{aligned}
$$

The "smoking gun" for gamma-ray production through the hadronic channel is therefore the accompanying production of secondary neutrinos.

VHE astronomy advanced significantly the last decade with telescope arrays like e.g. the High Energy Sterioscopic System (H.E.S.S. I and II) that is operational in Namibia, as well as VERITAS in the USA and MAGIC in the Canary Islands. For example, H.E.S.S. has made the most significant contribution towards unravelling the mysteries of the VHE gamma-ray universe since $65 \%$ (e.g. [5]) of the current sources in the online TeVCAT have been detected by H.E.S.S. (see e.g. http://tevcat.uchicago.edu/ or http://www.icrr.u-tokyo.ac.jp/ morim/TeV-catalog/) (see Fig. 2).

The advent of the Cherenkov Telescope Array (CTA), an even bigger international collaboration than H.E.S.S., namely a collaboration between 1200 scientists from 177 institutes and 28 countries, will make an even more significant impact in the field of VHE astronomy. The paper will be structured as follows: In the following section the main properties of CTA will be discussed briefly and compared with other high energy facilities like Fermi-LAT, Magic and H.E.S.S.. This will be followed by a general discussion of particle acceleration in cosmic environments, especially focussing on spun-up white dwarfs in close binaries, with the emphasis on AE Aquarii and the newly discovered AR Scorpii. This will be followed by conclusions. 


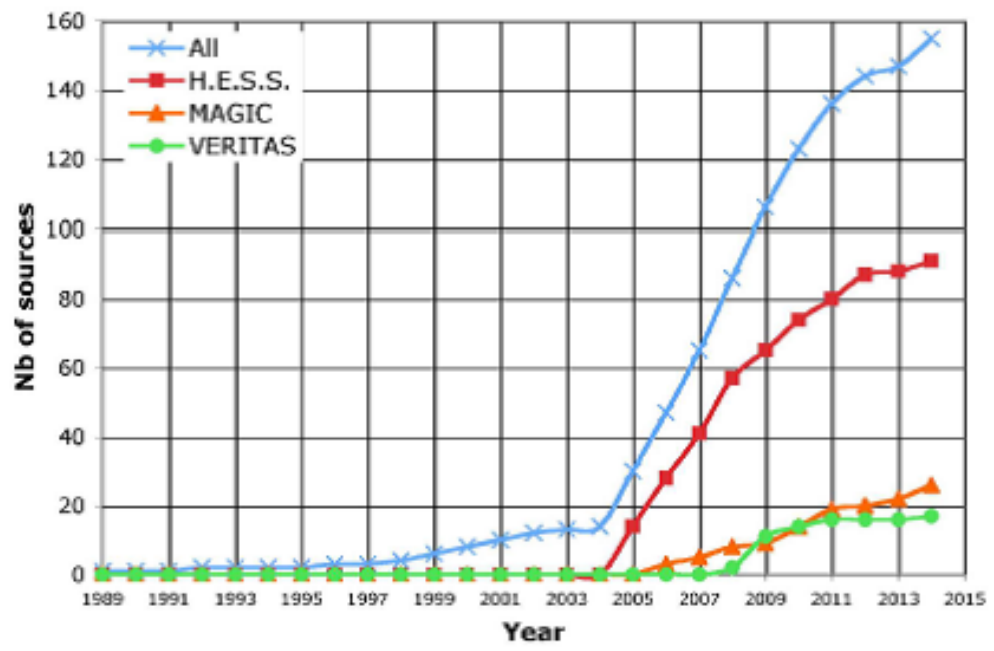

Figure 2: The detection of VHE gamma-ray sources since 1989 illustrating the contribution of H.E.S.S towards unravelling the mysteries of the high energy universe. Adopted from [5].

\section{The Cherenkov Telescope Array (CTA): The first open VHE observatory}

The increase in sensitivity and resolving power that the Cherenkov Telescope Array (CTA) introduce will most probably revolutionize the field of VHE astronomy, resulting in new classes of VHE astronomical sources being discovered. The CTA will consist of two nodes, a northern node with telescopes placed at La Palma, the Canary Islands (Spain), and the southern node with telescopes placed in Paranal (Chile). Back-up sites are Aar (Namibia) and San Pedro Mártir (Mexico) for the southern and northern nodes respectively (see Fig. 3). The CTA will be the first open Cherenkov observatory that will provide open time, where guest observers can apply for observation time, to supplement consortium time that will be devoted to core programs and surveys.

The site selection and telescope hardware were optimized to lower the threshold energy to math-up CTA with Fermi-LAT in the mid-GeV range. For example, the threshold energy for an atmospheric Cherenkov telescope is given by

$$
E_{\mathrm{T}} \propto \frac{1}{C(\lambda)} \sqrt{\frac{B(\lambda) \Omega \tau}{\eta(\lambda) A(\lambda)}}
$$

with $C(\lambda), B(\lambda), \Omega, \tau, \eta(\lambda)$ and $A(\lambda)$ representing the Cherenkov flux produced by incoming gamma-rays (or protons) in the atmosphere (altitude dependent), sky brightness, opening solid angle of the telescope, integration time of electronics, quantum efficiency of photomultiplier tubes and collection area of telescope respectively. The large collection area of CTA, compared to other gamma-ray facilities, combined with advances in detector technology, among other factors, will reduce the threshold energy and increase the sensitivity of CTA in the mid-GeV energy range to levels significantly above Fermi-LAT and the current generation of Air Cherenkov Telescopes. Lower threshold energies allow CTA to make a significant contribution towards the study of gamma- 


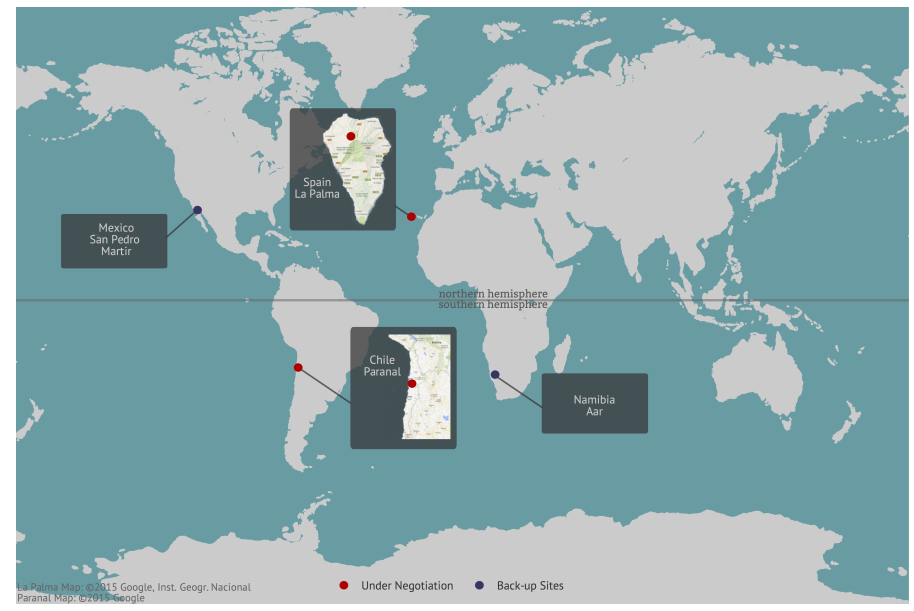

Figure 3: Paranal (Chile) and La Palma (Canary Islands) are the chosen sites for the southern and northern nodes of CTA, with back-up sites Aar (Namibia) and San Pedro Mártir (Mexico). Adopted from https://portal.cta-observatory.org/Pages/Preparatory-Phase.aspx

ray pulsars detected by Fermi-LAT in the GeV energy range, as well as to avoid the absorbing effect due to pair production (e.g. [6]) as a result of the interaction between high energy gammarays and lower energy photons, allowing the study of the gamma-ray universe to higher redshifts (e.g. [7, 8, 9]). The redshift horizons for CTA in the planned energy range have been determined (e.g. [10] and references therein) for an extra-galactic background light model proposed by ([11]) (see Fig. 4). It can be seen (Fig. 4) that the universe is transparent $(\tau \leq 1)[\tau$ represents the optical depth] for gamma-ray photons with energy up to $\sim 50 \mathrm{TeV}$ to redshifts of $z \sim 10^{-2}$, and for higher redshifts $(z \geq 1)$ the universe is transparent $(\tau \leq 1)$ to gamma-rays with energies $\leq 50 \mathrm{GeV}$.

The CTA array (e.g. [12]) will consist of $\sim 100$ telescopes of three different sizes to optimize its sensitivity over a wide energy range from $\sim 50 \mathrm{GeV}$ to $\sim 100 \mathrm{TeV}$, namely a low energy array consisting of a few 20-30 m class telescopes, a core array consisting of several 10-12 m class telescopes and a high energy array consisting of a larger number of 5-6 m class telescopes spread over a larger area $\left(\sim\right.$ few $\left.\mathrm{km}^{2}\right)$. The comparative sensitivity and the angular resolution of CTA compared to other high energy facilities are presented in Fig. 5 and Fig. 6 respectively (e.g. [12]). The total field of view of the telescope array will be greater than $8^{\circ}$. The benefits of its superior sensitivity, large field of view and excellent angular resolution can be summarized as follows:

\section{Sensitivity:}

- Access VHE populations across the entire Galaxy

- Sample fast variability in various classes of sources (e.g. AGN, GRB)

Field of View $(\mathrm{FoV})>8^{\circ}$ :

- Study diffuse regions

- Efficient and rapid survey of large fields 


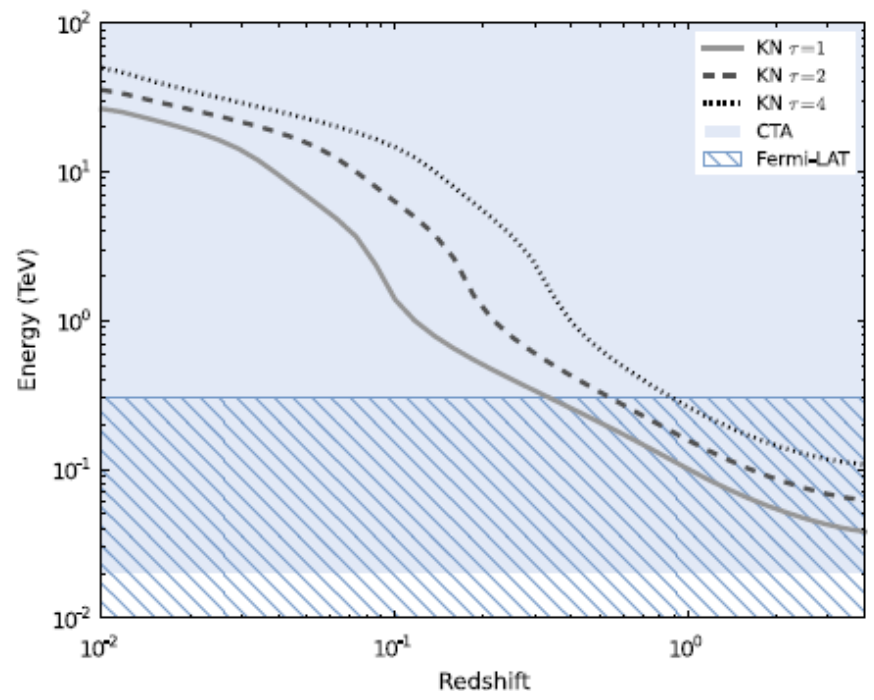

Figure 4: The comparative redshift horizons for CTA and Fermi-LAT as a function of energy for gamma-ray interactions with predicted limits of extra-galactic background light. Adopted from [10].

\section{Arcminute Angular Resolution:}

- Resolve extended sources (SNR, starbursts)

\section{Broad Energy Coverage:}

$-<100 \mathrm{GeV}$ to reach larger redshifts

- $>10 \mathrm{TeV}$ to search for PeVatrons

In the discussion above it has been clearly shown that CTA will introduce a "paradigm shift" concerning sensitivity and angular resolution, while its wide field of view will make gamma-ray surveys possible. For example, the $\sim$ few arcminute resolution at energies around $\sim 10 \mathrm{TeV}$ may provide the required resolution to isolate the PeVatron source that was reported recently by the H.E.S.S. collaboration coinciding with the position of Sag A* (e.g. [13]), as well as other high energy sources in densely populated fields of the galactic centre and Magellanic clouds.

It has been shown that the CTA will be a "game changer" in the field of high energy astrophysics, with superior sensitivity and angular resolution, it will most probably add significantly to the legacy of H.E.S.S., which already produced a "bonanza" (e.g. [4]) of high energy gamma-ray sources. In the next section a brief discussion of particle acceleration in compact objects will be presented, focussing only on the acceleration in rotating magnetized compact objects. The role of shock acceleration in magnetized cosmic plasmas has been reviewed recently (e.g. [3] and extensive references therein) and fall outside the scope of this study.

\section{Cosmic Accelerators: Rotating Magnets}

It can be shown (e.g. [14]) that electric and magnetic fields in a reference frame co-moving (primed quantities) with a highly conducting fluid or plasma transform as follows to the laboratory, 


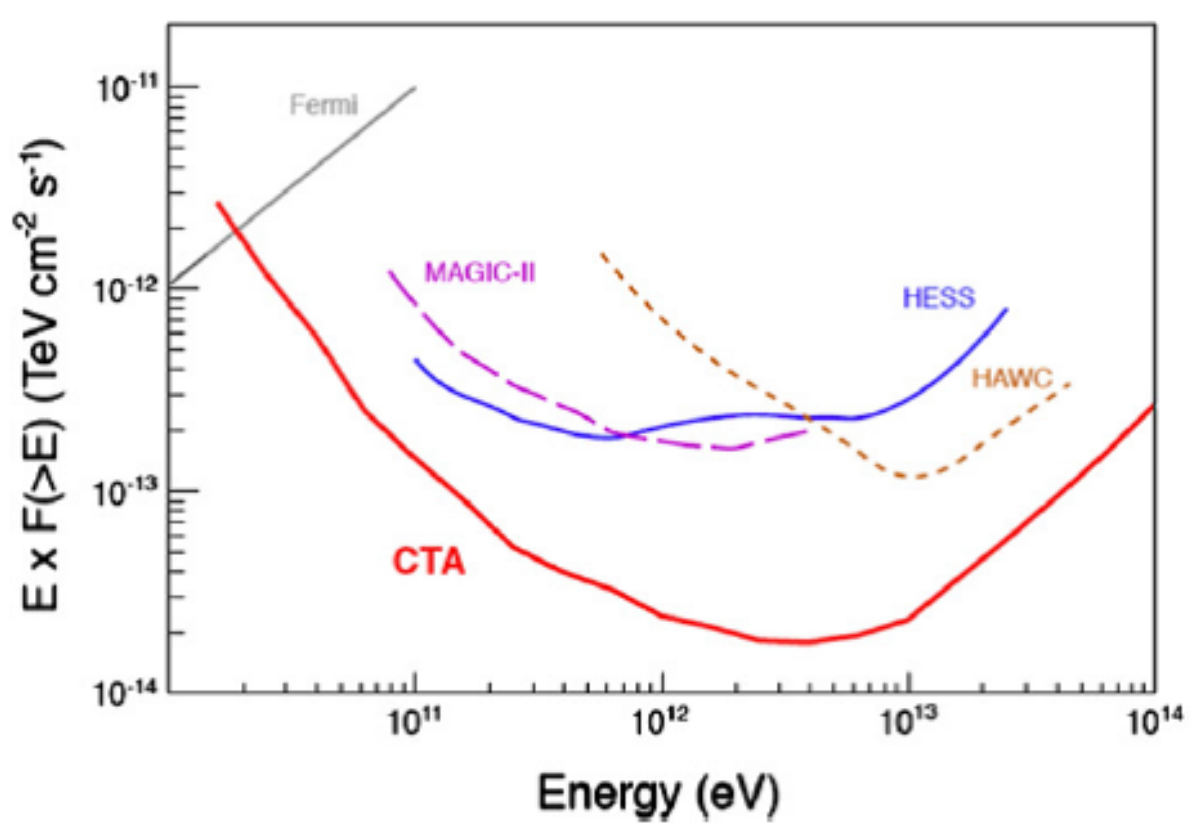

Figure 5: The sensitivity curve of CTA compared to other high energy facilities. Adopted form [12].

i.e. the observer's reference frame (unprimed quantities):

$$
\begin{aligned}
& \mathbf{E}=-\Gamma\left(\beta \times \mathbf{B}^{\prime}\right) \\
& \mathbf{B}=\Gamma \mathbf{B}^{\prime},
\end{aligned}
$$

where $\beta=\frac{\mathbf{v}}{c}$ and $\Gamma$ represents the Lorentz factor. The maximum energy that can be attained by a charged particle of charge $Z e$ is

$$
\begin{aligned}
\varepsilon_{\max } & =Z e \int \mathbf{E} . \mathbf{d s} \\
& =q \beta \Gamma R_{\mathrm{s}} B^{\prime},
\end{aligned}
$$

where $R_{\mathrm{S}}$ and $B^{\prime}$ representing the size of the source and magnetic field in the frame of the source. In the fast rotating magnetosphere of a compact object like a neutron star or white dwarf, these velocity-related parameters (i.e. $\beta \Gamma$ ) are associated with the rotational velocity of the source with respect to a stationary observer. This expression clearly illustrates that for fast rotating compact objects, satisfying $(\beta \Gamma>>1)$, the rotational kinetic energy reservoir relaxes the requirements for the strength of the intrinsic magnetic fields in the source to accelerate charged particles to high energies. It can be seen from Figure 7 that even white dwarfs, with magnetic fields ranging between $\sim 10^{5} \mathrm{G}-10^{8} \mathrm{G}$, can theoretically accelerate charged particles to energies of the order of $\varepsilon_{\max } \sim 10^{20} \mathrm{eV}[15]$.

\section{Spun-up White Dwarfs in Close Binaries}

A characteristic property of neutron star pulsars powered by rotational energy loss instead of accretion of matter is the ratio $\alpha=L_{\mathrm{x}} / L_{\mathrm{s}-\mathrm{d}} \sim 10^{-3}$ (e.g. [16]) of X-ray luminosity to spin-down 


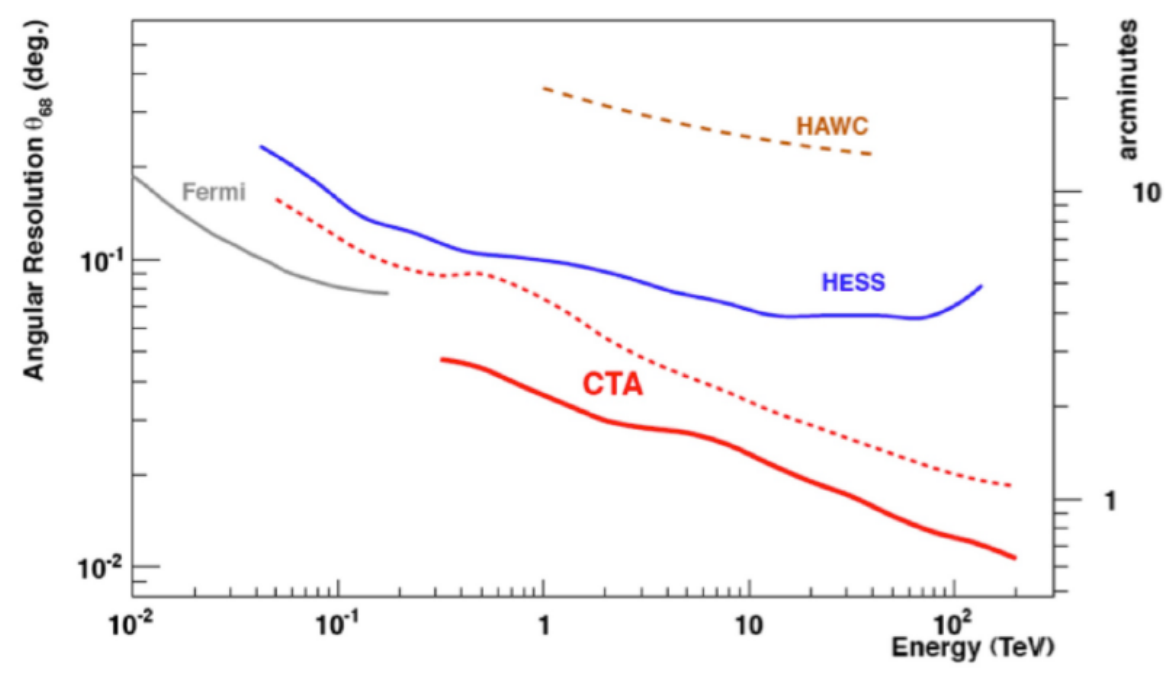

Figure 6: The angular resolution of CTA compared to other high energy facilities. The solid line provides the angular resolution of CTA obtained from events with ten or more images, while the dashed line shows the angular resolution for events with only two images. Adopted form [12].

power of the white dwarf (see Fig. 8). Some fast rotating magnetized white dwarf systems, for example the nova-like variable AE Aquarii which is currently in a propeller state (e.g. $[17,18,19])$, exhibits a similar ratio of X-ray luminosity to spin-down power $\left(\alpha=L_{\mathrm{X}} / L_{\mathrm{S}-\mathrm{d}} \sim 10^{-3}\right)$ as the spin-powered pulsars (e.g. [16]). This may provide the right conditions for pulsar-like particle acceleration and resultant gamma-ray production in these systems through leptonic and hadronic channels. This opens-up the possibility of a new class of potential high energy source, i.e. spun-up white dwarfs in close binary systems, that can be explored with CTA.

It has been shown (e.g. [20,21]) that the nova-like variable AE Aquarii may have evolved from a run-away high mass transfer history, during which the white dwarf was spun-up to its current short rotation period. During this run-away mass transfer phase the white dwarf accreted roughly at the Eddington limit, resulting in nuclear burning to have taken place on the surface of the white dwarf. It has been shown [20] that for AE Aquarii, the white dwarf could have been spun-up to a period around $33 \mathrm{~s}$ in a time scale of roughly $t_{\mathrm{s}-\mathrm{u}} \sim$ few $\times 10^{4} \mathrm{yr}$, which resulted in the white dwarf to have entered the propeller phase when the high mass transfer subsided. During the current propeller phase, the white dwarf will be in a state of low mass accretion, resulting in a tenuous pulsar-like magnetosphere which can sustain high potential differences between the polar cap and equator (e.g. [22, 23]) that can accelerate charged particles to energies in excess of 10 $\mathrm{TeV}$ outside the light cylinder of the rapidly rotating white dwarf. It has been shown (e.g. [23]) that relativistic electrons with Lorentz factors of $\gamma_{\mathrm{e}} \sim 10^{7}$ can up-scatter soft photons from the secondary companion to energies of the order of

$$
\varepsilon_{\gamma} \leq 20\left(\frac{\gamma_{\mathrm{e}}}{10^{7}}\right)^{2}\left(\frac{\varepsilon_{\mathrm{ph}}}{0.05 \mathrm{eV}}\right) \mathrm{TeV}
$$




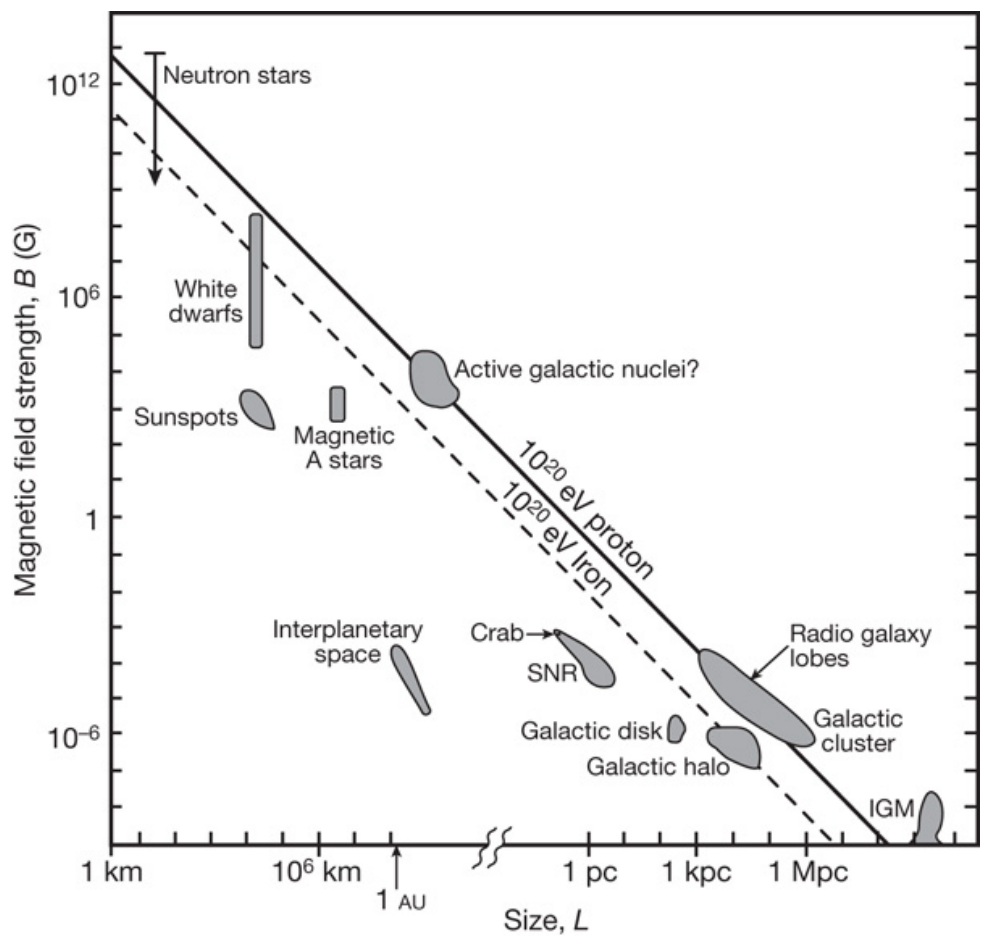

Figure 7: The Hillas Diagram. On the horizontal axis there is the typical size of the source and the vertical axis gives the typical source magnetic field. The diagonal lines correspond to the requirements for a $10^{20} \mathrm{eV}$ proton and heavy nucleus respectively. Adopted from [15].

in the Thomson limit. From an energetics point of view this provides interesting possibilities for VHE emission from spun-up white dwarfs in binary systems. This also provides substance to the reports of burst-like VHE gamma-ray emission from AE Aquarii in the 1990s (e.g. [24, 25, 26]) by two independent groups respectively. It has to be mentioned that during a more recent multiwavelength campaign ([27]) AE Aquarii was observed for 12 hours by the MAGIC group during which time the source displayed moderate transient emission in optical and X-ray wavelengths. However, during this campaign no pulsed VHE gamma-ray emission above $200 \mathrm{GeV}$ was detected. These authors (i.e. [27]) deduced an upper limit for the VHE power above $200 \mathrm{GeV}$ up to 3 orders of magnitude lower than the inferred burst-like power reported by $([24,26])$. It has to be mentioned that the burst-like VHE events reported by $([24,26])$ correspond to a duty cycle of only $0.024 \%$ over the total 280 hours of observing time stretching over 4 years (e.g. [26]), which implies that these events may be quite rare and positive detection requires continuous or regular monitoring in the VHE gamma-ray energy regime.

Recently another spun-up white dwarf system, AR Scorpii (AR Sco), has been discovered by the Warwick group [28]. This system also shows a spun-up white dwarf with a rotation period of the order of 2 minutes in a 3.56 hour binary system. The white dwarf in AR Sco, like AE Aquarii, show an $\alpha=\left(L_{\mathrm{x}} / L_{\mathrm{s}-\mathrm{d}}\right) \sim 10^{-3}$ ratio for the X-ray luminosity to spin-down power (see Fig. 8), similar to spin-powered neutron star pulsars [16]. For AR Sco, the white dwarf field is estimated to be $B_{*} \leq 500 \mathrm{MG}$, assuming that the spin-down of the white dwarf is purely due to the pulsar-like 


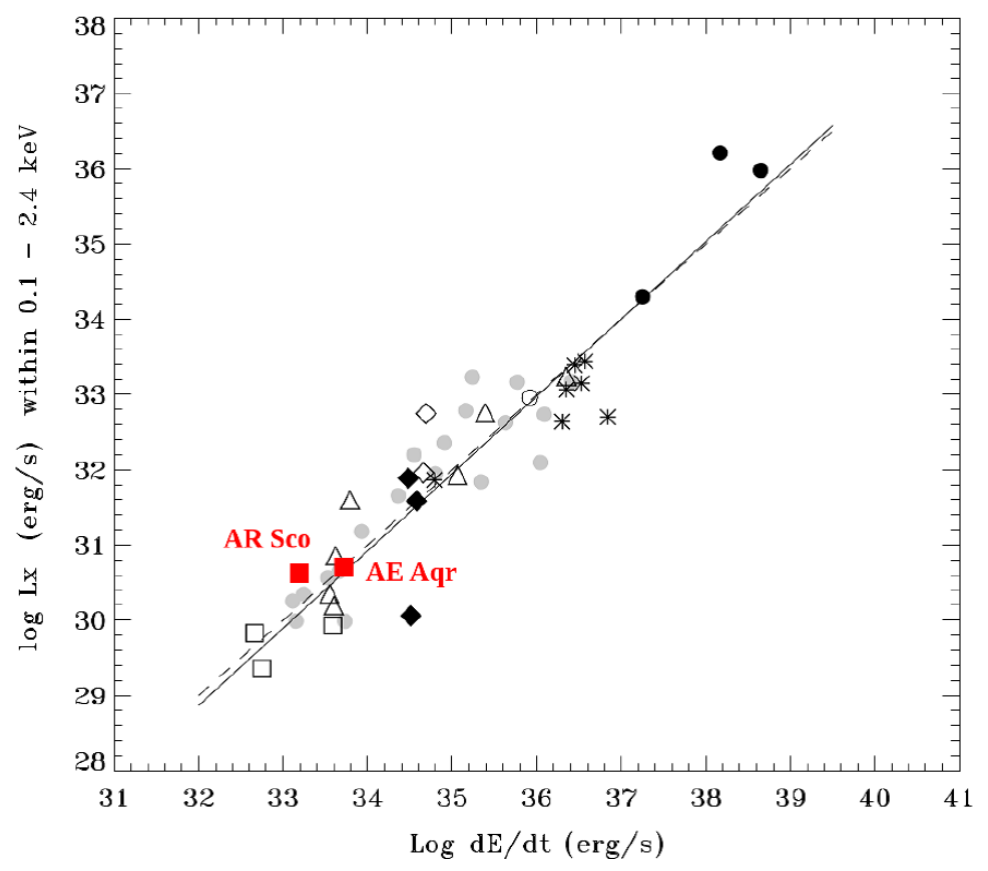

Figure 8: The accretion power vs spin-down luminosity of several spin-powered neutron stars. Included are two spin-powered white dwarf pulsar systems, AE Aquarii and AR Scorpii. The emission in both of these systems may be driven totally by the spin-down power of a highly spun-up magnetized white dwarf. Adapted from [16].

magnetic dipole radiation from the spinning magnetosphere [28]. From an energetics point of view this poses interesting possibilities for the possible acceleration of particles to relativistic energies and subsequent emission and detection of VHE gamma-rays given its proximity of $d \sim 100 \mathrm{pc}$, similar to AE Aquarii.

\section{Discussion}

The advent of CTA, with its unprecedented sensitivity and resolution in the energy regime between $50 \mathrm{GeV}$ and $100 \mathrm{TeV}$, combined with large survey projects e.g. like meerKAT (in radio) and in optical, the Large Synoptic Survey Telescope (LSST), which will probably discover thousands of new radio and optical variables, will really open-up new horizons in the field of high energy emission from various classes of galactic and extra galactic systems. This will certainly introduce new surprises related to VHE gamma-ray emission from various classes of compact sources in different astrophysical environments

\section{Acknowlegements}

The author thank the organisers for the invitation to the conference and their hospitality, as well as an anonymous referee for the critical assessment of the paper and a number of contructive suggestions that significantly improve the impact of the paper. 


\section{References}

[1] J. Cronin, T. Gaisser \& S. Swordy, Origin and Propagation of Cosmic rays, Sci. Amer. 276 (1997) 44

[2] Auger Collaboration, Update on the correlation of the highest energy cosmic rays with nearby extragalactic matter, Astropart. Phys. 34 (2010) 314

[3] A.R. Bell, Cosmic ray Acceleration, Astropart. Phys. 43 (2013) 56

[4] E. Lorenz \& R. Wagner, Very High Energy Gamma-Ray Astronomy, arXiv:1207.6003v1[physics.hist-ph] 25 July 2012

[5] B Degrange \& G. Fontaine, Introduction to high energy gamma-ray astronomy, $C . R$. Physique 16 (2015) 587-599

[6] M.S. Longair, High Energy Astrophysics (2nd edition), Vol. 1, Cambridge University Press, Cambridge, (1992) 128

[7] R.J. Gould \& G. Schréder, Opacity of the universe to high energy photons, Phys. Rev. Lett. 16 (1966) 252

[8] J.V. Jelley, High energy gamma-ray absorption in space by a $3.5 \mathrm{~K}$ microwave field, Phys. Rev. Lett 16 (1966) 479

[9] G.G. Fazio \& F.W. Stecker, Predicted high energy break in the isotropic gamma-ray spectrum, a test of cosmological origin, Nature 226 (1970) 135

[10] D. Mazin et.al., Potential of EBL and cosmological studies with the Cerenkov telescope Array, Astropart. Phys., 43 (2013) 241

[11] T.M. Kneiske \& H. Dole, A lower limit flux for extragalactic background light, Astronomy \& Astrophysics, 515 (2010) A19

[12] B.S. Acharya et al., Introducing the CTA Concept, Astropart. Phys., 43 (2013) 3

[13] The H.E.S.S. Collaboration, Acceleration of petaelectronvolt protons in the galactic centre, Nature 531 (2016) 476

[14] J.D. Jackson, Classical Electrodynamics (2nd ed), Wiley \& Sons, New York, (1975) 473, 552

[15] P.M. Bauleo \& J.R. Martino, The dawn of the particle astronomy era in ultra-high energy cosmic sources, Nature 458 (2009) 847

[16] W. Becker \& J. Trümper, The x-ray luminosity of rotation powered neutron stars, A\&A 326 (1997) 682

[17] M. Eracleous \& K. Horne, A speedy magnetic propeller in the cataclysmic variable AE Aquarii, ApJ 471 (1996) 427 
[18] G.A. Wynn, A.R. King \& K. Horne, A magnetic propeller in the cataclysmic variable AE Aquarii, MNRAS 286 (1997) 436

[19] P.J. Meintjes \& L.A. Venter, The diamagnetic blob propeller in AE Aquarii and the nonthermal radio to mid-infrared emission, MNRAS 360 (2005) 573

[20] P.J. Meintjes On the evolution of the novalike variable AE Aquarii, MNRAS 336 (2002) 265

[21] K. Schenker, A.R. King, U. Kolb, G.A. Wynn \& Z. Zhang AE Aquarii:how cataclysmic variables descend from supersoft binaries, MNRAS 337 (2002) 1105

[22] N.K. Ikhsanov \& P.L. Biermann High energy emission from fast rotating white dwarfs, A\&A 445 (2006) 305

[23] B. Oruru \& P.J. Meintjes, X-ray characteristics and the spectral energy distribution of AE Aquarii, MNRAS 421 (2012) 1557

[24] C.C.G. Bowden et al., 350 GeV gamma-rays from AE Aquarii, Astropart. Phys. 1 (1992) 47

[25] P.J. Meintjes et al., AE Aquarii: An emitter of pulsed TeV gamma-rays resembling optical emission during flares, ApJ 401 (1992) 325

[26] P.J. Meintjes et al., Simultaneous optical and TeV gamma-ray observations of the cataclysmic variable AE Aquarii, ApJ 434 (1994) 292

[27] J. Aleksić et al., MAGIC search for VHE $\gamma$-ray emission from AE Aquarii in multi-wavelength context, A\&A $\mathbf{5 6 8}$ (2014) A109

[28] T.R. Marsh et al., A radio pulsing white dwarf binary star, Nature, 537 (2016) 374 